\title{
http://jaet.journals.ekb.eg \\ EPILEPTIC SEIZURE DETECTION IN IOHT: A VISUAL IMAGE-BASED PROCESSING APPROACH
}

\author{
Ali A. Khalil ${ }^{1, *}$, Ashraf A. M. Khalaf ${ }^{1}$, Ghada El-Banby ${ }^{2}$, Turky Al-Otaiby ${ }^{3}$, Saleh Al-Shebeili ${ }^{3}$, Fathi E. Abd El \\ - Samie $^{2}$ \\ ${ }^{1}$ Communications and Electronics Engineering Department, Faculty of Engineering, Minia University, Egypt \\ ${ }^{2}$ Department of Electronics and Electrical Communications Engineering, Faculty of Electronic Engineering, Menoufia \\ University, Menouf 32952, Egypt \\ ${ }^{3}$ KACST, Dept. of Electrical Engineering, King Saud University, Riyadh, KSA. \\ * Corresponding author E-mail: ali_wee@yahoo.com
}

\begin{abstract}
This paper presents a new technique for electroencephalography (EEG) seizure detection from multi-channel EEG signals based on image processing concepts in Internet-of-Health-Things (IoHT) systems. The multi-channel EEG segments are treated as two-dimensional matrices as if they were images. Scale-space analysis with Scale Invariant Feature Transform (SIFT) is used to extract the feature points in the up-mentioned two-dimensional matrices. The number of points is used as a discriminating factor between seizure segments and normal segments. An exhaustive study of the 24 patients of the Children's Hospital Boston (CHB-MIT) database is presented in this paper. The EEG signals are transmitted via WiFi/Bluetooth, then all their signals are segmented into one-second segments, the numbers of features points are extracted from these segments, the Probability Density Function (PDF) of the number of feature points for normal and seizure segments are estimated. The Equal Error Rate (EER) is estimated between PDFs of the numbers of feature points in seizure and normal segments. Simulation results on all patients reveal the ability of the proposed technique to set a patient-specific discrimination threshold of $70 \%$ of Max spectral power for seizure detection with an accuracy of $95.6 \%$.
\end{abstract}

Keywords: Electroencephalography (EEG); Scale Invariant Feature Transform (SIFT); IoHT; Epileptic Seizure detection; Visual image processing.

\section{INTRODUCTION}

Many definitions of "epilepsy" in the literature exist; at least there are four different proposals of the International League Against Epilepsy (ILAE) referring to "epilepsy". The most recent definition of epilepsy is "Epilepsy is a disorder of the brain characterized by an enduring predisposition to generate epileptic seizures and by the neurobiological, cognitive, psychological, and social consequences of this condition" [1-4]. Epilepsy has the same meaning as "seizure disorders". It is worth noting that epilepsy ranks fourth in terms of the most common neurological diseases. Epilepsy has many types affecting different ages and causing many health problems [5]. A seizure is caused by sudden, abnormal, and excessive electrical activity in a group of brain cells. Seizures start when the nerve cells send wrong or distorted signals that ignite patients with distressing feelings, which makes them acting strangely, usually as a spasm or a violent vibration involving their muscles $[6,7]$.

Seizures accompanied by temporary symptoms. These symptoms include loss of consciousness or awareness, disturbances of movement, loss of sensation or cognitive functions such as vision, hearing and taste, and others. It varies from one patient to another, it depends on the location where the brains' disturbance first starts, how long

Received: 29 June, 2021, Accepted:14 October, 2021 it continues, and how far it spreads. The ILAE released a new classification of seizures in 2017, it depends on clinical causes rather than pathogenic cases; the new classification consists of three classes: Focal onset, Generalized onset, Unknown onset. A Focal seizure is a seizure that begins in an area or groups of cells in one brain side. A focal aware seizure or simple partial seizure is a seizure that happens during awake and aware. It will have called focal impaired awareness seizure or complex partial seizure if it happens during confusion or unconsciousness. Generalized onset seizures are seizures that happen when areas or group of cells on both brain sides are affected. An unknown onset seizure is the seizure that has an unknown location of the beginning, or the seizures have no available information, it may be diagnosed as a focal or generalized when more information is gathered later [8-11].

Although there is no cure for epilepsy permanently, it can be controlled largely by medicines, or by using smart tools. Otherwise, surgical intervention is required immediately. According to WHO, up to $70 \%$ of seizure cases can be completely controlled successfully with antiepileptic drugs (AEDs). However, in some cases when seizures last for long periods, or when it is repeated at high rates without consciousness in between, it is diagnosed as a medical emergency. 


\section{Vol.42, No.1. January2023}

Applications of computer vision and artificial intelligence became very touchable and widely used. The field of smart health care has developed tremendously, accompanied by the development of many emerging technologies, especially in the field of the Internet-ofThings (IoT). The Internet-of-Health-Things (IoHT) has emerged as an effective way to save the lives of individuals in many diseases, especially epilepsy cases. Many seizure detection algorithms and methods are developed in IoHT; it is a smart system that consists of sensors, transmitters, network medium, processor, storage unit, and application [12]. The paper includes significant novel technique as it presents applying a new proposed approach for effective seizure detection using the SIFT algorithm on IoHT systems, making use of its provided highly discriminative feature descriptor. The proposed approach using SIFT algorithm succeeded efficiently in seizure detection, it achieved low EER and FAR.

\section{Literature Review}

The analysis of human brain activity is very important for better understanding and diagnosing related diseases. Seizure duration usually lasts from thirty seconds to two minutes in most cases, doctors use brain scans and other diagonal tests including blood tests and medical history to detect and diagnose seizures. The electroencephalogram (EEG) is a detecting process of the brain's electrical activity using an electrical measuring device called electrodes. The electrode is a flat and small metal tool, connected to the brain scalp. EEG is one of the main diagnostic measures for epilepsy. The brain cells send electrical signals continuously. These signals are classified to the delta, theta, alpha, beta, and gamma according to their frequency range, which spans from $0.1 \mathrm{~Hz}$ to more than $100 \mathrm{~Hz}$. EEG records are the main source for investigating seizure epilepsy; EEG records are still rich and have more information to be revealed, it has a big amount of valuable data [13].

Most algorithms of seizure detection have two main stages; first, features extraction of EEG brain records through an appropriate quantitative method. Next, a classification criterion is applied; this criterion may be a modern machine-learning algorithm or a simply applied threshold that differentiates seizures from normal intervals [14- 16]. A survey of EEG seizure detection techniques based on the processing domain was presented by Alotaiby et al. (2014). They classified seizure detection methods to timedomain methods, frequencydomain detection algorithms, wavelet domain, Empirical Mode Decomposition (EMD), Principal Component Analysis (PCA), Singular Value Decomposition (SVD), and Independent Component Analysis (ICA) domain. Most detection techniques adopt time-domain or wavelet-domain algorithms [17]. Massoud, et al. (2019) introduced a comparison of detection techniques for human feature points, the comparison shows that the Laplacian of Gaussian (LoG) technique has the most quality [18].

Dey, et al. (2017) proposed a reliable ECG monitoring system, they developed a low cost and improved Zigbee wireless unit [20]. Daoud, et al. (2018) introduced a seizure detection system using EMD with Deep Neural Network in [21]. Park, et a. (2018) presented a seizure detection model using Spatio-temporal correlation and Convolution Neural Network in [22]. Kaur (2021) proposed a wireless body area network using Castalia Simulator in [19], the system relied on a medium access control layer and was characterized as low cost and low power consumption. Sundaravavidel, et al. (2018) developed a low-cost nutrition monitoring system using the deep learning method in [23]. Yuan, et al. (2018) introduced a seizure detection system using wavelet-transform context fusion in [24]. Sayeed, et al. (2019) introduced an accurate seizure detection system using machine learning, they developed an Edge-Device for seizure detection [25, 26]. Dolgin, (2020) introduced a seizure detecting smartwatch that alerts healthcare providers in [27]. Olokodana, et al. (2020) presented a realtime automatic seizure detection system using DWT with the Kriging model in [28].

This paper proposes a new model using the Scale Invariant Feature Transform (SIFT) algorithm in a visual image-based manner; the SIFT algorithm is applied on transmitted EEG records to identify and distinguish the time of disturbances from normal intervals.

\section{Methodology}

Our proposed system consists of a "cap" that collects EEG signals, the cap is supported by a group of compact, ultra-low power sensor that collects data. The collected EEG signal is transmitted via Wifi/Bluetooth to a smartphone application or any wearable device "watch". Next, a feature extraction stage of EEG segmented frames, a threshold-based classification process, and finally, the application sends the data for a continuous monitoring system at emergency vehicles, smart hospitals, family members, or any healthcare service provider, as shown in Fig. (1). The processing stage uses the sift algorithm, which is an AI system that detects the EEG status, takes decisions, sends information to those involved, and updates records.

As shown in Fig. (2). First, the transmitted signals are

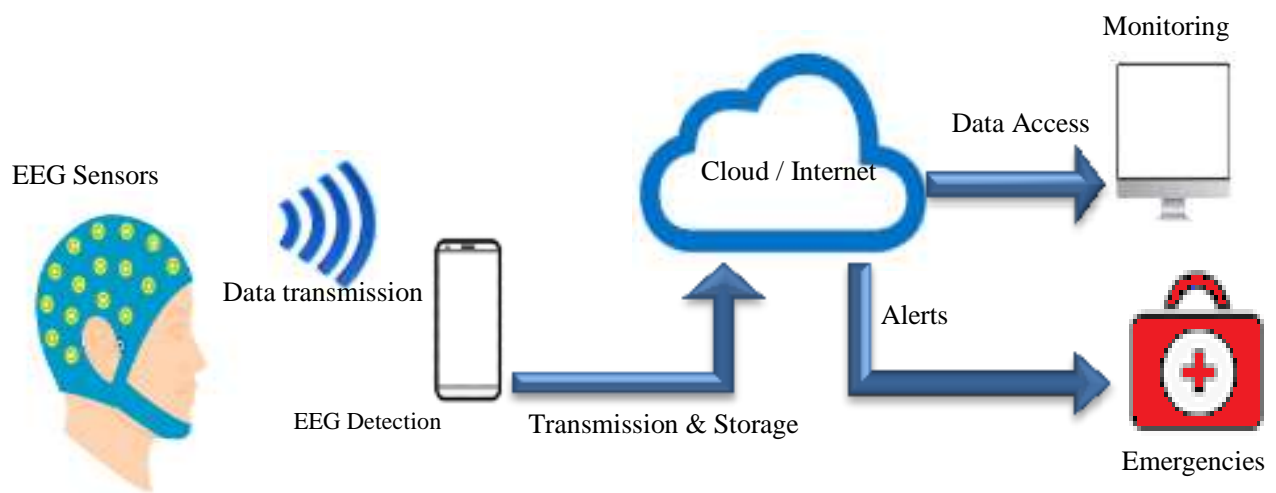

Fig. 1. The proposed architecture

sent via Bluetooth to the mobile application. Next, the signal is segmented into fixed time length windows, the windowing process output is a segmented signal, which is 
Vol.42, No.1. January2023

treated as an image, it represents features of seizure time. The SIFT is used as a feature descriptor for images, it extracts abstract features from a local point in the image; these features are extracted to represent the information around this point by representing the information in a features vector or features descriptor. The approach is based on using the SIFT to detect regions of seizures in the segmented frame of EEG signal and differentiate these periods from the normal signals. Decisions are taken according to an applied

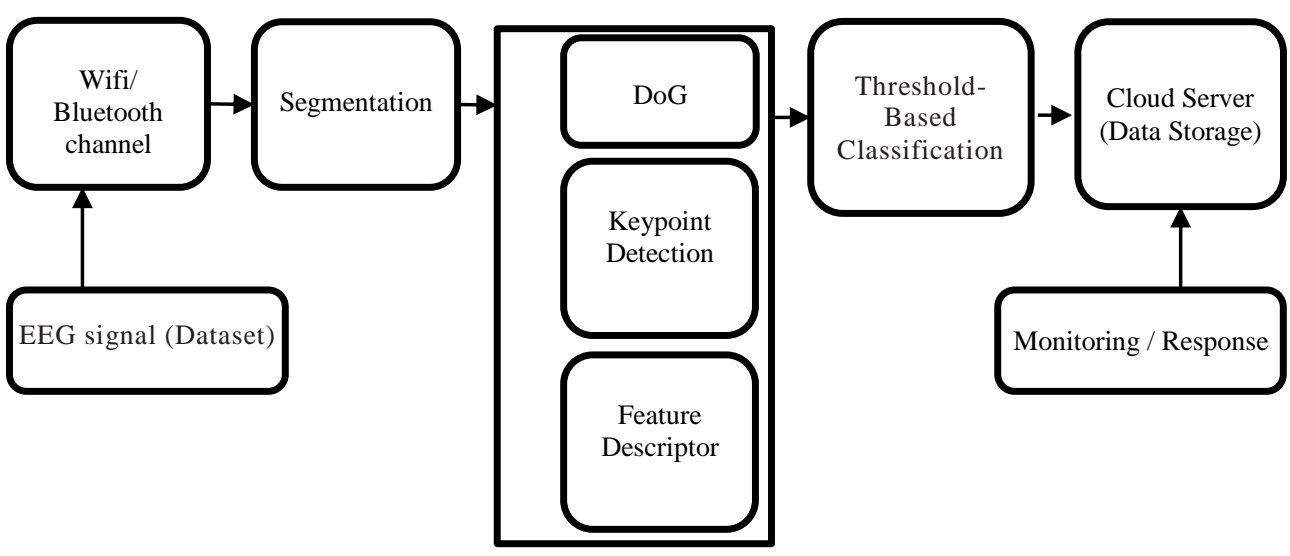
threshold, then the data is sent to a cloud server where records are updated, and a monitoring system is watched. This is a very cost-effective solution where cloud-based monitoring systems are also available.

The objective of using SIFT for features representation is to produce a highly discriminative signature of the seizure signal, which experimented as a local image trying to make it more distinctive as possible from normal brain signals. In the proposed approach SIFT algorithm encodes interesting information in the brain signal into a series of numbers and act as a sort of numerical point "fingerprint". Consequently, the numerical point is used to differentiate one signal feature from another. Finally, this information is used to detect seizure time; these intervals could be easily differentiated from the normal periods. The approach makes use of SIFT advantages for feature description.

\section{a. Scale-Invariant Feature Transform algorithm}

(SIFT)

The SIFT algorithm was published by David Lowe, University of British Columbia Canada in 1999; SIFT extracts features in a feature descriptor whose features remains unchanged when the transformation is applied. It remains invariant to changes in scale, illumination, blur, and local affine distortions. Besides, it is easy to extract, highly distinctive, and has a low error probability when used for object identification. SIFT includes both feature detection and feature description, SIFT only refers to feature descriptor, while the term "SIFT key point" is usually used to indicate the detected feature. Fig. (3) shows the most popular and frequently used image feature descriptors. There are many other techniques, but not shown here for space limitation [29-30].

In the proposed approach, SIFT algorithm is applied to the segmented frames, the number of interest keypoint is calculated using the Difference of Gaussians (DoG), a feature vector is composed for each keypoint using a scale, orientation, and location parameters. The local orientation of the image is calculated over several scales and over neighborhoods around the point of interest using the local image properties to provide invariance against rotation. Finally, the feature descriptor is calculated

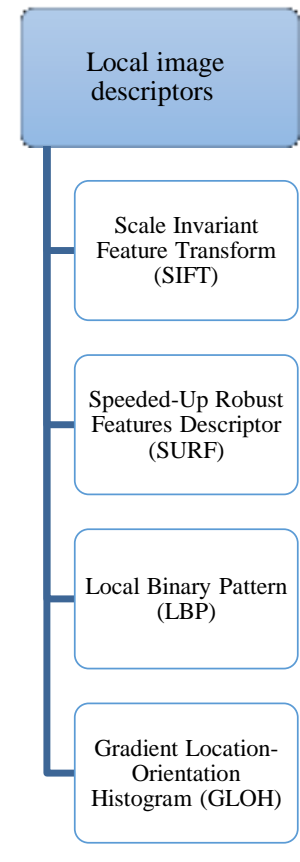

based on the detected information of each key point.

\section{i. Scale-Space Representation}

SIFT operates on a Gaussian scale-space to achieve scale invariance, this is achieved by convolving the original image with gradually increased Gaussian variances. The Gaussian convolution of the input image $\mathrm{I}(\mathrm{x}, \mathrm{y})$ with Gaussian blur $\mathrm{G}(\mathrm{x}, \mathrm{y}, \mathrm{k} \sigma)$ at scale $\mathrm{k} \sigma(\sigma$ is the standard variance); $\mathrm{D}(\mathrm{x}, \mathrm{y}, \mathrm{k} \sigma)$ calculated as:

$$
\mathrm{D}(\mathrm{x}, \mathrm{y}, \sigma)=\mathrm{G}(\mathrm{x}, \mathrm{y}, \mathrm{k} \sigma) \times \mathrm{I}(\mathrm{x}, \mathrm{y})
$$

Thus, a set of convolved images $\{\mathrm{G}(\mathrm{x}, \mathrm{y}, \sigma \mathrm{i}), \mathrm{i}=1,2, \ldots, \mathrm{n}\}$ can be used to denote the Gaussian scale space of the original image I, $\sigma$ is increased gradually by a constant factor $\mathrm{k}, \sigma \mathrm{i}+1=\mathrm{k}$ - $\mathrm{i}$. SIFT takes the extrema in the DoG scale space as the initial keypoint, to be robust to scale changes and with high repeatability. To achieve this, the DoG scale space is constructed by subtracting adjacent scales of the image:

$$
\operatorname{Ds}(\mathrm{x}, \mathrm{y}, \sigma)=\mathrm{D}(\mathrm{x}, \mathrm{y}, \mathrm{k} \sigma)-\mathrm{D}(\mathrm{x}, \mathrm{y}, \sigma)
$$

Thus, to apply Difference of Gaussians (DoG) function to the series of smoothed and resampled images in scale space, a DoG image $\operatorname{DG}(\mathrm{x}, \mathrm{y}, \sigma)$ is:

$$
\operatorname{DG}(\mathrm{x}, \mathrm{y}, \sigma)=\operatorname{Ds}(\mathrm{x}, \mathrm{y}, \mathrm{ki} \sigma)-\operatorname{Ds}(\mathrm{x}, \mathrm{y}, \mathrm{kj} \sigma)
$$
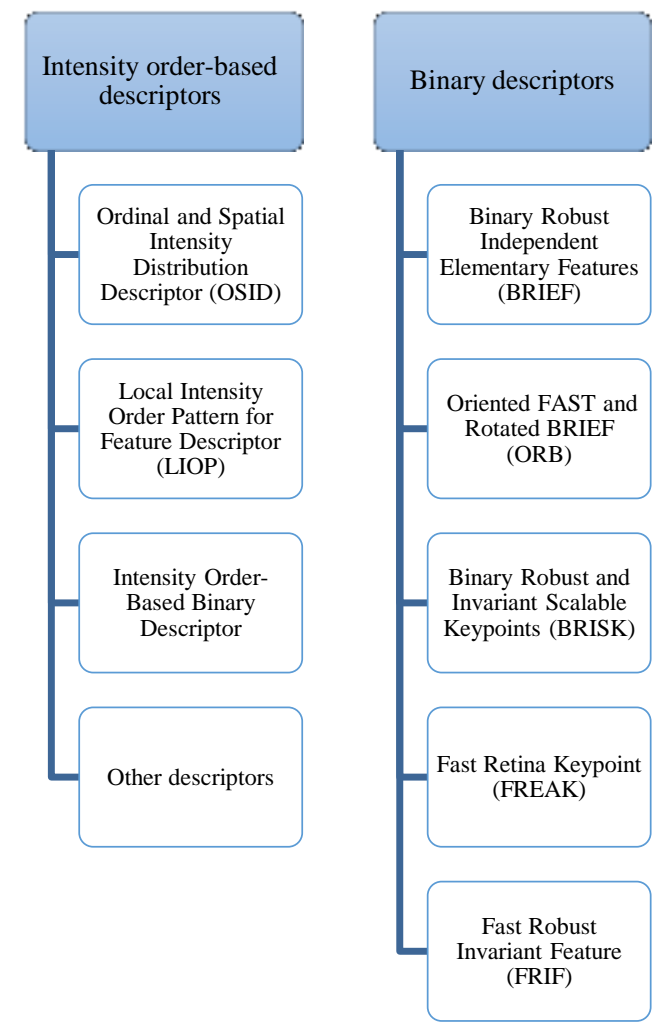

Fig. 3: The popular image feature descriptors. 


\section{ii. Keypoint features selection}

The maxima and the minima of the result define the key locations. Low contrast and edge points are discarded to achieve recognition stability and better matching. These key points are stored to be indexed, and the high probability nearest neighbours' points are identified using the Best-binfirst $(\mathrm{BBF})$ algorithm. Each pixel in the DoG images is compared to its eight neighbours at the same scale and 18 corresponding neighbouring pixels in the above and below neighbouring layer (nine in each of them).

The close and unstable local extrema are suppressed using non-maximum suppression, (i.e. if the pixel value is the maximum or minimum among all compared pixels, it is selected as a candidate key point). This is a variation of one of the methods developed by Lindeberg [31]. The best-binfirst (BBF) algorithm limits searching after the first 200 nearest neighbour candidates, this helps speed up search and results in no more than 5\% loss of correct matches while eliminating $90 \%$ of false matches. Hessian matrix $\mathrm{H}$ is computed at the key point location and scale [32]:

$$
H=\left[\begin{array}{ll}
D_{x x} & D_{x y} \\
D_{y x} & D_{y y}
\end{array}\right]
$$

\section{iii. Features Selection Mechanism}

The SIFT algorithm begins by sampling the magnitudes and orientations of the image gradient in a region of $16 \times 16$ around each key point, a local descriptor is constructed to describe the key point in a scale and rotation-invariant manner using each detected keypoint parameter including position, scale, and orientation [33,34]. The local region defined by the above parameters is divided into $4 \times 4$ grids sub-region that contains eight orientation pins in each as shown in Fig. (4), to compute histograms of gradient orientations in these grids.

Finally, to form a set of orientation histograms, all histograms are concatenated together to form a 128dimensional descriptor $(4 \times 4$ histograms each with eight bins, producing a feature vector of $4 \times 4 \times 8=128$ element per keypoint). These concatenated histograms are normalized to gain invariance to affine changes in illumination as a final step producing a unit vector as the SIFT descriptor [35].
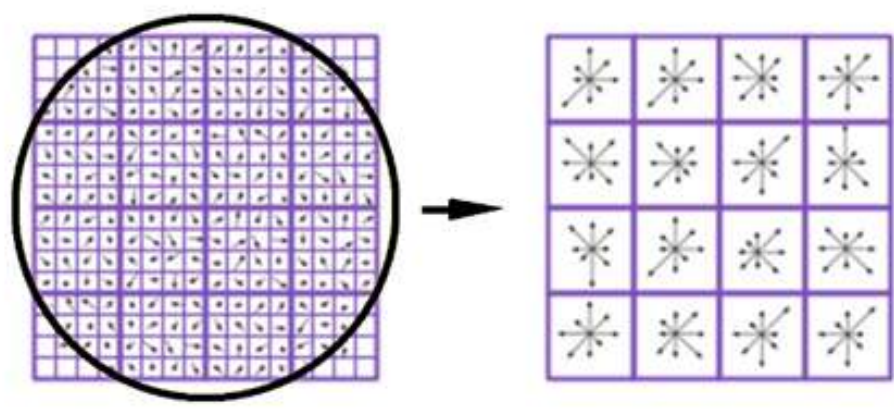

Fig. 4: Mechanism of the SIFT features for a $16 \times 16$ pixel and a $4 \times 4$ array.

The methodology of the proposed approach can be detailed as follows: First, EEG records are transmitted via Bluetooth. Next, EEG signals of multi-channel natures are segmented into one-second segments, taking every 256 samples as one segment (window size of one second) to obtain the spectral features in this period.

After obtaining the spectral features, DoG is calculated to construct a scale-space for each segment. Then, applying the Hessian matrix to detect the keypoints, and estimate the number of feature points. The next step is the estimation of PDFs for No. of feature points across signals Finally, the estimation of the discrimination threshold and equal error rate (EER).

An electroencephalographic scalp database containing brain records was used for different patients with epileptic seizures; the database was recorded at the Children's Hospital Boston (CHB-MIT) database, available online [36] The records duration is more than 40 hours per most cases, it is divided into an hour for recording. It shows abnormalities of brain signals before, during, and after these episodes. The data files saved in the European Data Format (EDF format) were captured in 23 channels with a sampling rate of $256 \mathrm{~Hz}$, the detailed information shown in Table 1.

Table.1: Detailed statistics extracted from CHB-MIT database (Values in seconds)

\begin{tabular}{|c|c|c|c|c|}
\hline $\begin{array}{c}\text { Patient } \\
\text { ID }\end{array}$ & N & $\begin{array}{c}\text { Total } \\
\text { duration }\end{array}$ & $\begin{array}{c}\text { Min. } \\
\text { Duration }\end{array}$ & $\begin{array}{c}\text { Max. } \\
\text { duration }\end{array}$ \\
\hline Chb01 & 7 & 442 & 27 & 101 \\
\hline Chb02 & 3 & 172 & 9 & 82 \\
\hline Chb03 & 7 & 402 & 47 & 69 \\
\hline Chb04 & 4 & 378 & 49 & 116 \\
\hline Chb05 & 5 & 558 & 96 & 120 \\
\hline Chb06 & 10 & 153 & 12 & 20 \\
\hline Chb07 & 3 & 325 & 86 & 143 \\
\hline Chb08 & 5 & 919 & 134 & 264 \\
\hline Chb09 & 4 & 276 & 62 & 79 \\
\hline Chb10 & 7 & 447 & 35 & 89 \\
\hline Chb11 & 3 & 806 & 22 & 752 \\
\hline Chb12 & 40 & 1475 & 13 & 97 \\
\hline Chb13 & 12 & 535 & 17 & 70 \\
\hline Chb14 & 8 & 169 & 14 & 41 \\
\hline Chb15 & 20 & 1992 & 31 & 205 \\
\hline Chb16 & 10 & 84 & 6 & 14 \\
\hline Chb17 & 3 & 293 & 88 & 115 \\
\hline Chb18 & 6 & 317 & 30 & 68 \\
\hline Chb19 & 3 & 236 & 77 & 81 \\
\hline Chb20 & 8 & 294 & 29 & 49 \\
\hline Chb21 & 4 & 199 & 12 & 81 \\
\hline Chb22 & 3 & 204 & 58 & 74 \\
\hline Chb23 & 7 & 424 & 20 & 113 \\
\hline Chb24 & 16 & 511 & 16 & 70 \\
\hline
\end{tabular}

\section{Analysis}

Extensive simulation experiments have been performed for all patients, the SIFT algorithms were applied to the transmitted records. First, the segmentation process is achieved by applying a window size of one second, taking 256 samples per segment. The DoG is calculated to construct a scale-space for each segment. Next, the Hessian matrix is applied and the number of key points is calculated for each segment during the seizure epileptics periods; this number is compared to the number of key points during normal periods. The change in the signal component during epileptic seizures is quantified to provide useful information; the PDFs are calculated versus the number of 
Vol.42, No.1. January2023

SIFT points for both seizure epochs and normal segments. Receiver Operating Characteristic curves (ROC curve) are calculated, also. It is a plot of the true-positive rate against the false-positive rate for the different possible cutpoints of a test.

Table.2: FAR, FRR, and EER

\begin{tabular}{|c|c|c|c|c|}
\hline ID & L & FAR & FRR & EER \\
\hline Chb01 & 100 & $6.02 \mathrm{E}-04$ & 0.89 & 0.0036 \\
\hline Chb02 & 100 & 0.0076 & 0.9946 & $2.21 \mathrm{E}-04$ \\
\hline Chb03 & 100 & $1.46 \mathrm{E}-04$ & 0.9259 & 0.0018 \\
\hline Chb04 & 100 & $9.74 \mathrm{E}-04$ & 0.9831 & $6.45 \mathrm{E}-04$ \\
\hline Chb05 & 100 & $7.92 \mathrm{E}-05$ & 0.9628 & 0.0019 \\
\hline Chb06 & 100 & 0.0237 & 0.9783 & $1.20 \mathrm{E}-22$ \\
\hline Chb07 & 100 & 0.0110 & 0.9352 & $6.32 \mathrm{E}-04$ \\
\hline Chb08 & 100 & $6.16 \mathrm{E}-05$ & 0.9793 & 0.0013 \\
\hline Chb09 & 100 & 0.0254 & 0.9503 & 0.0019 \\
\hline Chb10 & 100 & 0.0136 & 0.8115 & 0.0044 \\
\hline Chb11 & 100 & 0.2706 & 0.9495 & $8.41 \mathrm{E}-04$ \\
\hline Chb12 & 100 & 0.0386 & 0.7382 & 0.0054 \\
\hline Chb13 & 100 & 0.0991 & 0.8563 & 0.0462 \\
\hline Chb14 & 100 & 0.0379 & 0.8655 & 0.0130 \\
\hline Chb15 & 100 & 0.0028 & 0.9647 & 0.0014 \\
\hline Chb16 & 100 & 0.0273 & 0.8277 & 0.0036 \\
\hline Chb17 & 100 & 0.0063 & 0.8738 & 0.0030 \\
\hline Chb18 & 100 & 0.0097 & 0.9195 & $2.76 \mathrm{E}-04$ \\
\hline Chb19 & 100 & 0.0219 & 0.9607 & 0.0017 \\
\hline Chb20 & 100 & 0.1800 & 0.8610 & 0.0662 \\
\hline Chb21 & 100 & 0.0294 & 0.8411 & 0.0043 \\
\hline Chb22 & 100 & 0.0220 & 0.9642 & 0.0010 \\
\hline Chb23 & 100 & 0.0048 & 0.8075 & 0.0033 \\
\hline
\end{tabular}

Fig. (5) to Fig. (9) show ROC curves for randomly selected patients, figures reveal the PDFs for No. of SIFT points for seizure and normal segments. For these, it is clear that a threshold estimation process is possible. Accuracy, Equal Error Rate (EER), False Accept Rate (FAR), and False Reject Rate (FRR) values are calculated as an evaluation criterion for a set of $\mathrm{L}$ points; results are tabulated in Table 2. As shown from statistics in Table 2, Low EER values reveal a high ability to detect seizures efficiently.

Table.3: Comparison of the existing seizure detection

\begin{tabular}{|c|c|c|}
\hline Work & Method & Accuracy \\
\hline Park et al. [7] & $\begin{array}{c}\text { Spatio tempral Correlation } \\
\text { \& CNN }\end{array}$ & $85.6 \%$ \\
\hline Yuan et al. [9] & WT-Ctx-Fusion & $95.7 \%$ \\
\hline $\begin{array}{c}\text { Olokodana et } \\
\text { al. [12] }\end{array}$ & \begin{tabular}{c} 
DWT with Kriging model \\
\hline $\begin{array}{c}\text { Current } \\
\text { approach }\end{array}$
\end{tabular} & $\begin{array}{c}\text { SIFT-based visual } \\
\text { approach }\end{array}$ \\
\hline
\end{tabular}

methods.

Accuracy of combined data $=95.60 \%$, Table 3 shows the accuracy comparison of the existing seizure detection methods for the same database; Park et al. achieved an accuracy of $85.6 \%$ by applying Spatiotemporal Correlation
\& CNN [7], Yuan et al. [9] used WT-Ctx-Fusion to achieve an accuracy of $95.7 \%$, and Olokodana et al. [12] used a DWT with Kriging model to achieve accuracy of $93.4 \%$. The proposed SIFT-based visual approach achieved accuracy of $95.6 \%$ with an applied threshold of $70 \%$; this is almost equal to the WT-Ctx-Fusion approach, which put the SIFT-based approach on the top set of recommended techniques in EEG detection in IoHT systems.

$$
\text { Accuracy }=\frac{\text { No. of accurately classified images }}{\text { Total } \text { No.of images }} \times 100
$$
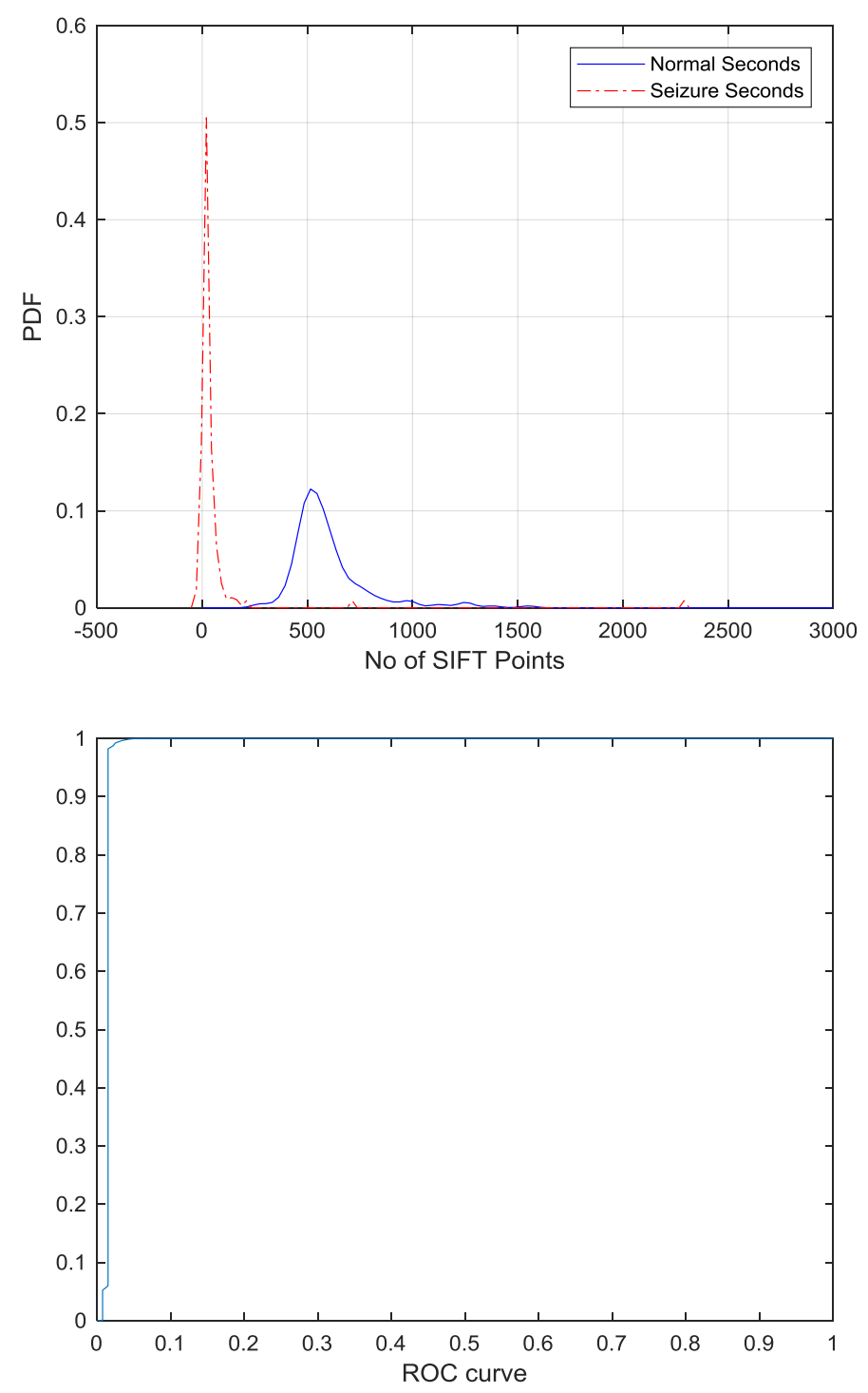

Fig. 5. Patient 2 and ROC curve. 
Vol.42, No.1. January 2023
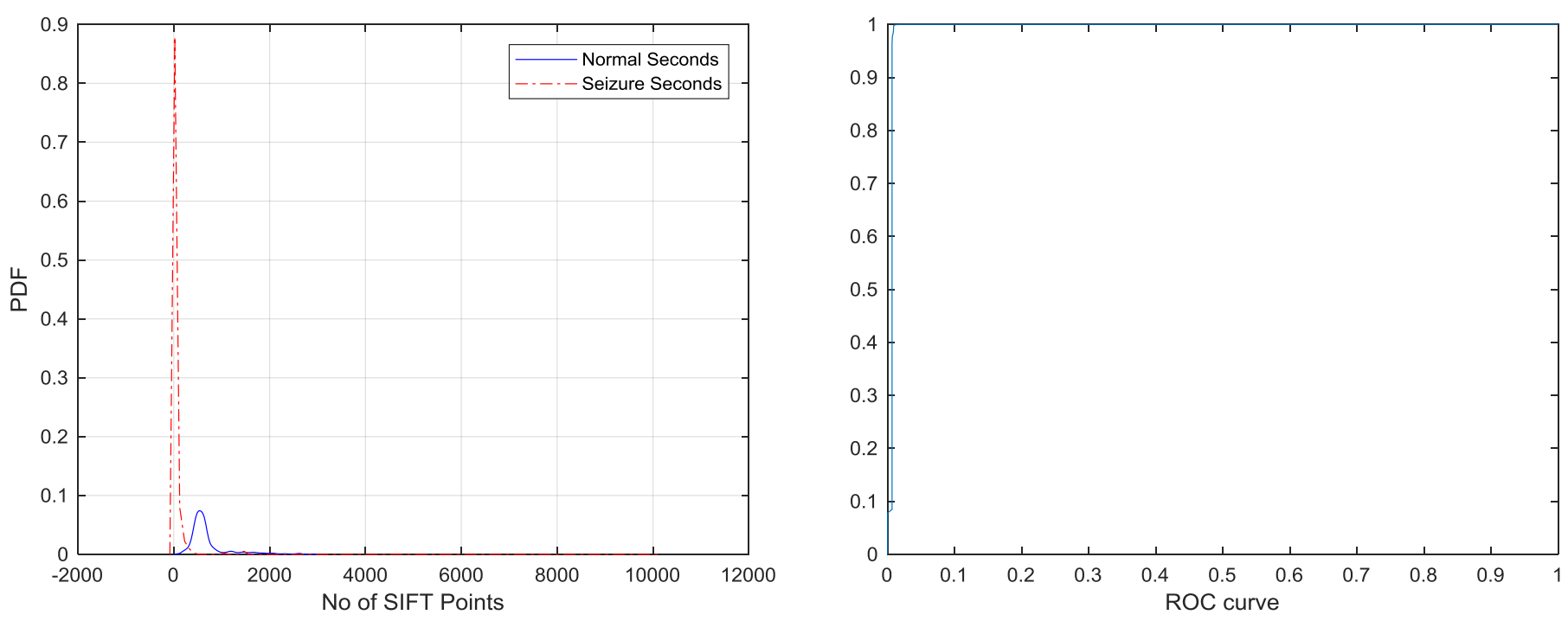

Fig. 7. Patient 5 and ROC curve.
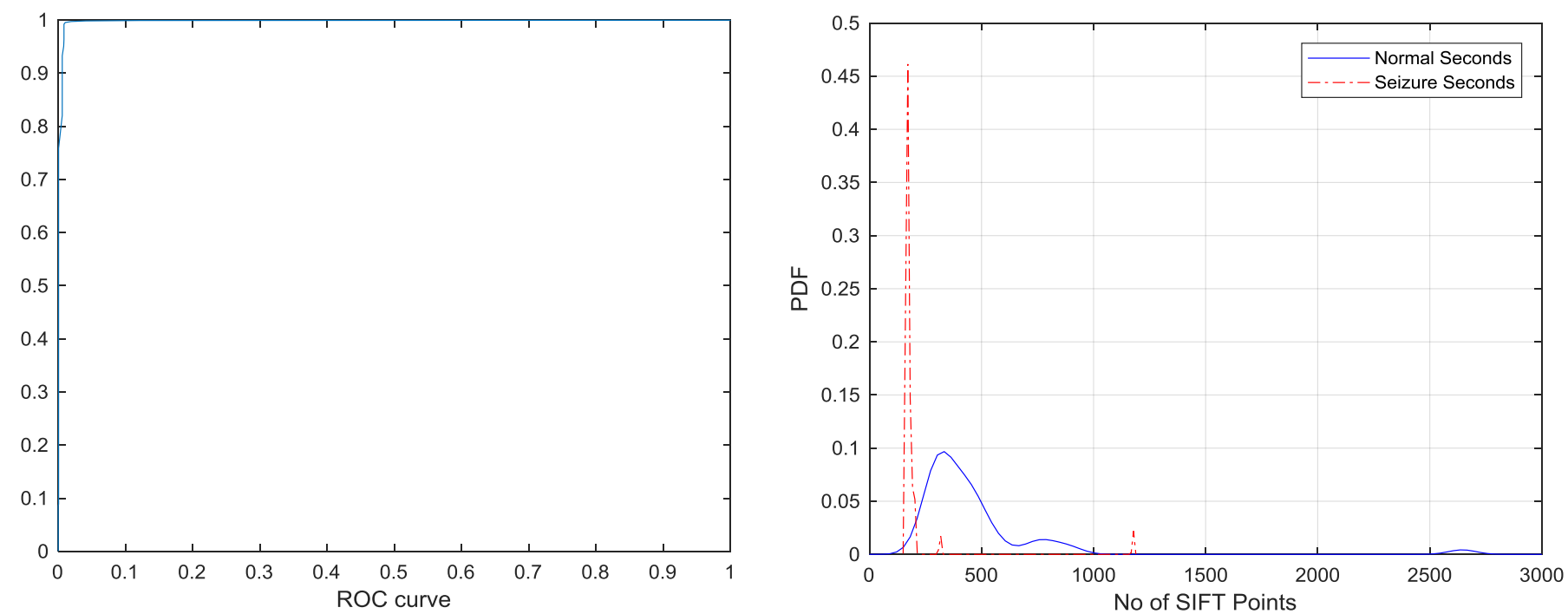

Fig. 6: Patient 3 and ROC curve.
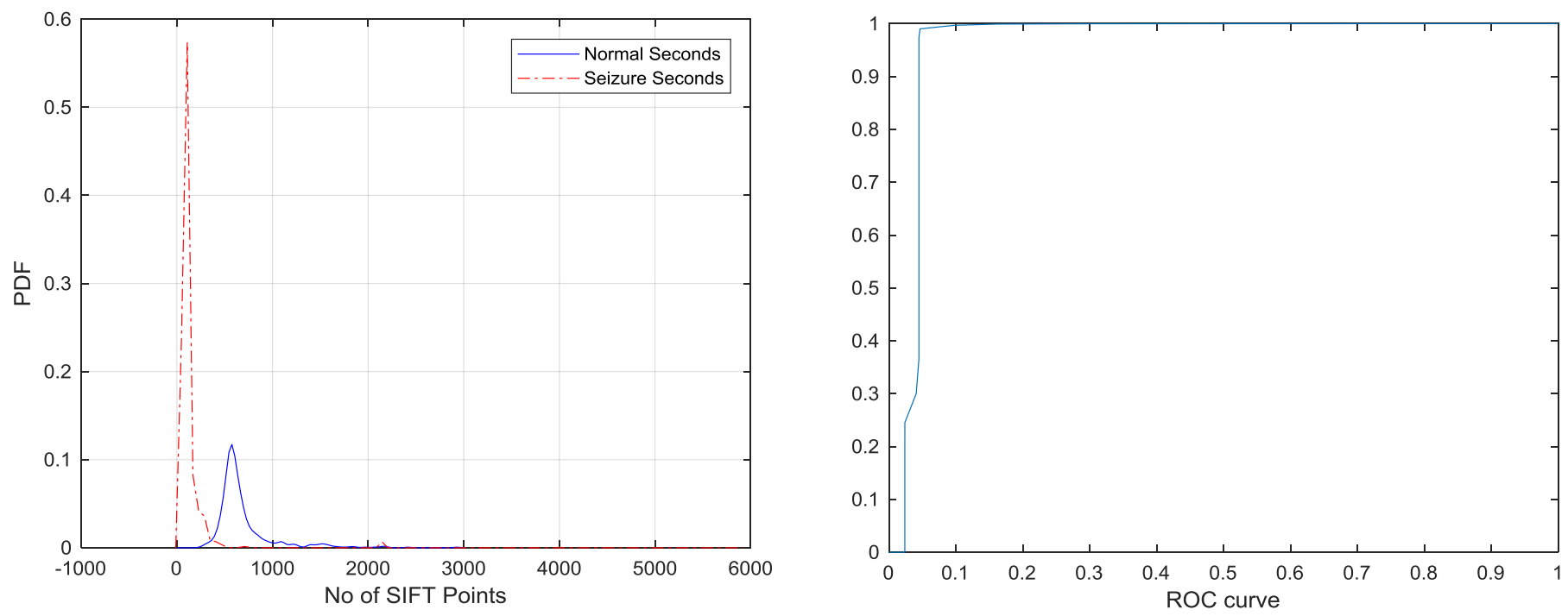

Fig. 8. Patient 6 and ROC curve. 
Vol.42, No.1. January2023
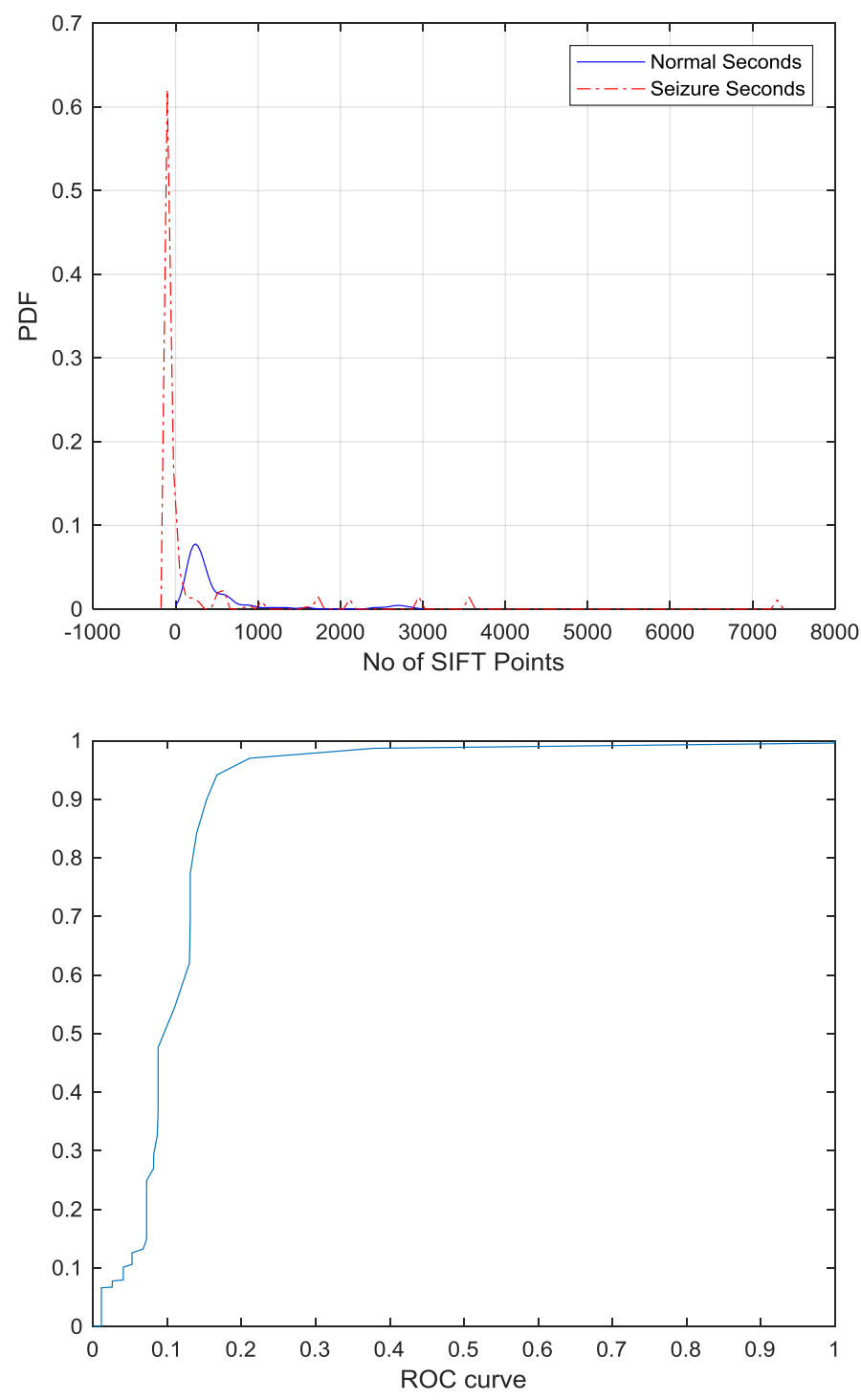

Fig. 9. Patient 7 and ROC curve.

\section{CONCLUSIONS}

This paper revealed that it is possible to deal with the multi-channel EEG transmitted segments in IoHT systems as if they are images. It is possible to apply 2 D feature extraction methods like SIFT to discriminate between seizure and normal activities during the seizure detection process. The proposed approach uses the SIFT descriptor algorithm adopted in image processing for an accurate visual representation of seizures. The basic idea of the approach is to represent the different activity levels of transmitted EEG signals as images. Next, the scale-space analysis is used to analyze these images for anomaly detection. The analysis is used to localize the seizures on the charts and represent EEG signal records. Experiments conducted with CHB-MIT database of 24 patients, 198 seizures, a total duration of approximately 3.23 hours, and more than 990 hours of recorded data. Simulation results revealed a high success rate for the detection of epileptic activities in the proposed approach. Results show that the number of SIFT points could be used easily to detect seizure intervals and differentiate them from normal brain signals making use of the provided highly discriminative feature descriptor. The proposed approach using the SIFT algorithm succeeded efficiently in seizure detection in IoHT systems achieving an accuracy of $95.6 \%$.

\section{REFERENCES}

[1] R. S. Fisher, C. Acevedo, A. Arzimanoglou, et al., ILAE Official Report: A practical clinical definition of epilepsy. Epilepsia. 55 (4), 475-82, 2014.

[2] S. Ashwal, Child Neurology: Its Origins, Founders, Growth and Evolution. 2nd ed. Academic Press Elsevier, 2021.

[3] R. V. Dodge, Grand Mal: A Life with Late Onset Epilepsy, McFarland, 187-193, 2020.

[4] J. Jessica, F. Walter, I. E. Scheffer, et al., The new definition and classification of seizures and epilepsy, Epilepsy Research. 139, 73-79, 2018.

[5] https://www.ilae.org/

[6] The World Health Organization: https://medlineplus.gov/epilepsy.html, Last access at 6-09-2021.

[7] Mayo Foundation for Medical Education and Research (MFMER): https://www.mayoclinic.org/testsprocedures/eeg/about/pac-20393875, Last access at 609-2021.

[8] http://www.who.int/mental_health/media/en/639.pdf

[9] R. Chang, C. Leung, C. Ho, et al., Classifications of seizures and epilepsies, where are we? A brief historical review and update, Journal of the Formosan Medical Association. 116(10), 736-741, 2017.

[10] https://clinicaltrials.gov/ct2/show/NCT02126774?term $=$ EPILEPSY\&rank=8, retrieved 6-9-2021.

[11] http://www.allcountries.org/health/epilepsy_historical _overview.html

[12] V. Suresh, J. Ramson, R. Jegan, Internet of Medical Things (IoMT) - An overview. 101-104, 2020.

[13] S. Shaban, O. Ucan, A. Duru, Classification of Lactate Level Using Resting-State EEG Measurement, Applied Bionics and Biomechanics, vol. 2021, 8 pages, 2021.

[14] P. Yash. Various epileptic seizure detection techniques using biomedical signals: a review. Brain Inf. 5, 6. 2018. 


\section{Vol.42, No.1. January2023}

[15] G. Giannakakis et al., Methods for Seizure Detection and Prediction: An Overview, 68, Springer Science Business Media, 2014.

[16] S. Ramgopal, S. Thome-Souza, M. Jackson, et al., Seizure detection, seizure prediction, and closed-loop warning systems in epilepsy. Epilepsy Behav. Epub 37:291-307, 2014.

[17] Alotaiby et al., EEG seizure detection and prediction algorithms: a survey, EURASIP Journal of Advances in Signal Processing, 83. 2014.

[18] M. A. Massoud, S. Kamal, comparison of edge detection techniques for human feature points extraction. Journal of Advanced Engineering Trends (JAET), 38(2), 121, 2019.

[19] M. Kaur, Performance and Scalability Evaluation of the Wireless Body Area Network using Castalia Simulator. Turkish Journal of Computer and Mathematics Education, 12(2), 543-554, 2021.

[20] N. Dey, A. Ashour, S. Fuqian, et al., Developing Residential Wireless Sensor Networks for ECG Healthcare Monitoring. IEEE Transactions on Consumer Electronics. 63. 10.1109/TCE. 2017.

[21] A. Abdelhameed, H. Daoud, M. Bayoumi, Deep Convolutional Bidirectional LSTM Recurrent Neural Network for Epileptic Seizure Detection. NEWCAS 10.1109, 2018.

[22] C. Park, G. Choi, J. Kim, et al., Epileptic seizure detection for multi-channel EEG with deep convolutional neural network. ELINFOCOM, 1-5. 10.23919, 2018.

[23] P. Sundaravadivel, K. Kesavan, L. Kesavan, et al., Smart-Log: A Deep-Learning Based Automated Nutrition Monitoring System in the IoT. IEEE Transactions on Consumer Electronics. PP. 1-1. 10.1109/TCE, 2018.

[24] Y. Yuan, G. Xun, K. Jia, et al., A multi-context learning approach for EEG epileptic seizure detection. BMC Systems Biology. 12. 10.1186/s12918-0180626-2, 2018.

[25] M. Sayeed, S Mohanty, E. Kougianos, et al., NeuroDetect: A Machine Learning Based Fast and Accurate Seizure Detection System in the IoMT. IEEE Transactions on Consumer Electronics. PP. 1-1. 10.1109/TCE, 2019

[26] M. Sayeed, S Mohanty, E. Kougianos, et al., eSeiz: An Edge-Device for Accurate Seizure Detection for Smart Healthcare. IEEE Transactions on Consumer Electronics. PP. 1-1. 10.1109/TCE, 2019.
[27] E. Dolgin, This seizure-detecting smartwatch could save your life. spectrum.ieee.org. 2018.

[28] I. Olokodana, S. Mohanty, E. Kougianos. OrdinaryKriging Based Real-Time Seizure Detection in an Edge Computing Paradigm. 10.1109/ICCE46568, 1-6. 2020.

[29] B. Fan, Z. Wang, F. Wu. Local Image Descriptor: Modern Approaches, Springer. Briefs in Computer Science, Springer-Verlag Berlin Heidelberg 2015.

[30] A. Awad,M. Hassaballah. Image Feature Detectors and Descriptors Foundations and Applications, Springer, 2016.

[31] T. Lindeberg, Image matching using generalized scale-space interest points". Journal of Mathematical Imaging and Vision, 52(1): 3-36, 2015.

[32] T. Lindeberg. Scale selection. In Computer Vision Springer, (2nd ed.) 2021.

[33] A. Awad, M. Hassaballah. Image Feature Detectors and Descriptors Foundations and Applications, Springer, 2016.

[34] M. Kashif,, T. M.,Deserno, D. Haak, et al. Feature description with SIFT, SURF, BRIEF, BRISK, or FREAK? A general question answered for bone age assessment. Computers in Biology and Medicine, 68, 67-75, 2016.

[35] J. Matas, O. Chum, M. Urban, et al. Robust widebaseline stereo from maximally stable extremal regions. Image and Vision Computing, 22(10), 761767. 2004

[36] CHB-MIT Scalp EEG Database, [Online]. Available: https://physionet.org/content/chbmit/1.0.0/. 
Vol.42, No.1. January2023

كثف نوبات الصرع في أنظمة انترنت الأثياء الصحية: طريقة معالجة قائمة على الصورة المرئية

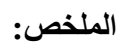

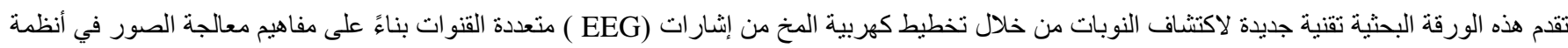

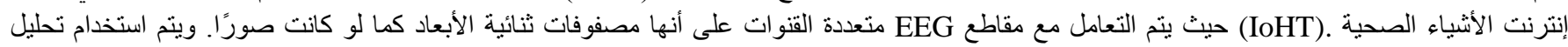

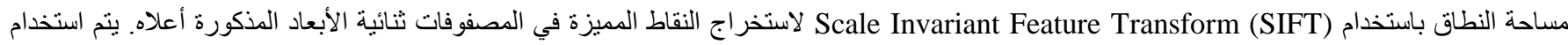

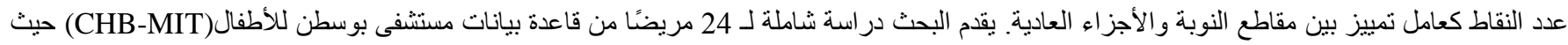

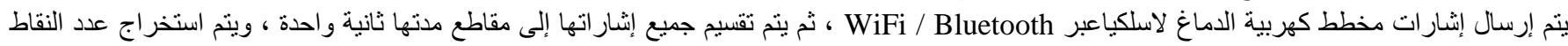

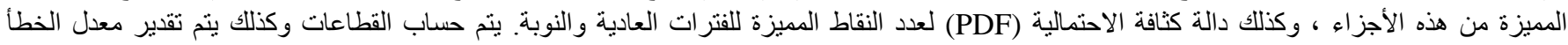

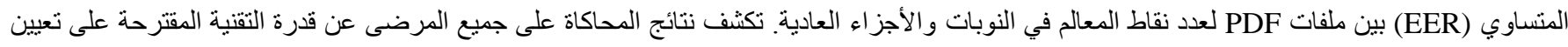

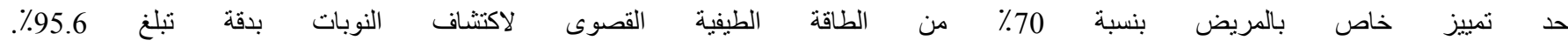

